\title{
Resource allocation for clustered network MIMO OFDMA systems
}

Jingya $\mathrm{Li}^{1 *}$, Carmen Botella ${ }^{2}$ and Tommy Svensson ${ }^{1}$

\begin{abstract}
In this article, we address the resource allocation problem for the downlink of a large network multiple input multiple output orthogonal frequency division multiplexing system with 3-sector base stations. The system is statically divided into a number of disjoint clusters of sectors. A two-step resource allocation scheme is proposed involving the inter-cluster and the intra-cluster levels. As a first step or inter-cluster level, two cooperative frequency reuse approaches are designed to mitigate the inter-cluster interference. A user partition method is proposed to divide the users of each cluster into cluster-edge and cluster-center users. To balance the cell-edge and the cellaverage performance, a fairness jug function is introduced to determine the frequency partition of the cooperative frequency reuse approaches. Then, as a second step or intra-cluster level, a utility-based joint scheduling and power allocation algorithm is proposed for each cluster, to maximize the sum utility of all users in the cluster under per-sector power constraints. Zero-forcing joint transmission is used across multiple sectors within the same cluster. Simulation results show that the proposed scheme can efficiently reduce the inter-cluster interference and provide considerable performance improvement in terms of both the cell-edge and cell-average user data rate. The proposed two-step resource allocation scheme can be implemented independently in each cluster without inter-cluster information exchange, which is an attractive property for practical systems, since it reduces both the network signaling overhead and the computational complexity.
\end{abstract}

Keywords: OFDMA, network MIMO, frequency reuse, resource allocation, interference coordination

\section{Introduction}

Driven by the demands to support data applications at higher throughput and spectral efficiency, orthogonal frequency division multiplexing (OFDM) based multiple access is being considered as a promising technique for the future wireless networks. OFDMA has been adopted as the downlink access technology of 3rd generation partnership project (3GPP) long term evolution (LTE) and LTE-advanced standards [1]. Based on the OFDM technique, OFDMA inherits the immunity to intra-cell interference. However, the inter-cell interference is still a major issue. In fact, a frequency reuse factor being equal to one causes serious inter-cell interference to users in the cell-edge areas, leading to poor cell-edge performance. Viable inter-cell interference mitigation approaches are reviewed in [2], including the use of

\footnotetext{
* Correspondence: jingya.li@chalmers.se

${ }^{1}$ Department of Signals and Systems, Chalmers University of Technology, Gothenburg, Sweden

Full list of author information is available at the end of the article
}

power control, fractional frequency reuse, opportunistic spectrum access, intra and inter-cell interference cancellation, and multiple input multiple output (MIMO) techniques.

Recently, coordinated multi-point transmission/reception (CoMP) has been proposed in 3GPP LTE-Advanced as a key technique to increase the system spectrum efficiency as well as the cell-edge performance [1]. In the case of CoMP joint transmission, both data and channel state information (CSI) of the users in CoMP mode can be shared by coordinated multiple cells, which can act as a single and distributed antenna array. Data to a user can be simultaneously transmitted from the coordinated cells to improve the received signal quality. Hence, the inter-cell interference is reduced by exploiting the signals transmitted from other cells to assist the transmission rather than treating them as interference. Notice that this technique is also referred as network coordination or network MIMO [3]. 
In a global network MIMO system, without any feedback, backhaul and synchronization constraints, the inter-cell interference can be completely eliminated. However, from a practical point of view, a major setback of global coordination is the large amount of feedback needed from the users and the large signaling overhead required for the inter-cell information exchange. An interesting tradeoff between the system performance and the required amount of CSI feedback and backhaul exchange has been pointed out [4-9]. This tradeoff is one of the reasons for restricting the use of network MIMO techniques to a limited number of cells or areas of the system. The system is typically divided into clusters of cells, and the joint transmission is implemented within the cells included in each cluster. The cluster formation can be static [10-14], or dynamic [15-17]. The static cluster formation specifies a predefined set of clusters of cells which do not change in time, whereas the dynamic clustering approaches form the clusters based on the varying channel conditions that users experience to different cells. Note that a coordinated cluster also causes inter-cluster interference to the users in the neighboring clusters, especially to users in the cluster-edge area. Therefore, the design of efficient inter-cluster interference coordination strategies and radio resource management algorithms is of great interest in the field of clustered network MIMO systems.

Previous studies about resource allocation in network MIMO OFDMA systems have mainly focused on the global network MIMO case [18-20], or on the single cluster case without considering inter-cluster interference [21-23]. Recently, research has shifted towards the limited coordination case with different cluster formation models, that is, resource allocation with dynamic clustering $[24,25]$ or resource allocation with static clustering [14]. In [24], an inter-cluster interference canceler performing linear processing on the downlink transmission signals is proposed for multi-user MIMO distributed antenna systems. However, a central unit $(\mathrm{CU})$ is needed to collect the global CSI of all users in the system and to calculate the transmission weight vectors for each cluster. Notice that this centralized framework requires an enormous amount of feedback and backhaul overhead. In [25], a centralized multi-cell network scheduling algorithm is proposed to minimize the inter-cluster interference by performing clustering from the user's point of view, which also needs a CU for global network scheduling. For a system with a large number of cells and a large number of users, a high computational burden will be caused in the CU. In [14], the authors instead consider a more realistic system model, where the network is divided into a number of disjoint static clusters, and limited inter-cluster coordination is used to pre-cancel interference for the users at the edge of neighboring clusters. In this approach, perfect CSI is available at the cluster side for both the cluster users and edge users in the neighboring clusters. Hence, each cluster can help the edge users in the neighboring clusters by taking these users into account when designing the precoding matrices. However, with a large number of users in each cluster, in order to serve a cluster-edge user, all the neighboring clusters need to provide a given number of degrees of freedom by dropping some scheduled users of their own, which leaves fewer degrees of freedom to serve their own users. In addition, a joint scheduling across clusters is needed for the whole network, which requires inter-cluster communication and increases the complexity of the resource allocation design.

Fractional frequency reuse is a promising technique for inter-cell interference mitigation. Instead of using spatial degrees of freedom to suppress the inter-cell interference, it restricts the available frequency resources of different cells through a predefined frequency reuse rule or through appropriate power control. In [26], the division of frequency resources is investigated for the uplink of a linear network MIMO system. Since the system is considered to be uniformly clustered in a linear grid, the inter-cluster interference can be completely eliminated with simple reuse strategies, e.g., half of the available frequency resources are assigned to each cluster with different resources assigned to adjacent clusters. In [27], the authors consider a more realistic scenario and employ appropriate power control in frequency such that adjacent clusters generate different interference levels in different subchannels. However, the power control problem is formulated in a centralized way such that a CU is needed for the network to solve the optimization problem for all the clusters. In [28,29], two frequency reuse schemes were proposed for a multi-cell OFDMA system, supporting non-coherent joint transmission to cell-edge users by user-centric dynamic clustering. Due to the user-centric nature of the clustering, these approaches also require a joint scheduling across cells for the whole network.

In this article, we address the resource allocation problem for the downlink of a clustered network MIMO OFDMA system with 3-sector base stations (BSs). Each sector has one directional antenna, and it is associated with a directional cell area. The whole system is statically divided into disjoint clusters of sectors. Due to practical issues (e.g., synchronization constraints, feedback constraints, backhaul network constraints and the system complexity), inter-cluster information exchange may not be feasible in realistic cellular systems. Targeting practical scenarios, radio resource allocation is independently performed in each cluster without intercluster communication. Zero-forcing beamforming is 
considered as the coherent joint transmission scheme within each cluster, which allows multiple users to share the same subchannel in each time slot by choosing proper beamforming weights. A two-step resource allocation scheme is proposed, which involves both intercluster and intra-cluster levels:

- As a first step or inter-cluster level of resource allocation, two novel cooperative frequency reuse approaches (CFR-1 and CFR-2) are proposed to mitigate the inter-cluster interference. A user partition method based on the long term channel gain is introduced to divide the users of each cluster into cluster-edge users (CEU) and cluster-center users (CCU). Frequency subchannels in each cluster are separated into two orthogonal sets, that is, clusteredge and cluster-center frequency sets. The intercluster interference is reduced by mapping different groups of CEU to different subchannels of the cluster-edge frequency set in a cooperative way. We also show that the frequency partition (the size of the cluster-center frequency set) and the user partition threshold are the parameters that can be optimized to balance the cell-edge and cell-average performance.

- As a second step or intra-cluster level of resource allocation, a sub-optimal utility-based joint scheduling and power allocation algorithm is proposed for each cluster with a low complexity. Assume that perfect CSI is available at the cluster side for the users within this cluster. The algorithm jointly determines the set of users scheduled on each subchannel, and the power allocation across subchannels. The objective is to maximize the sum utility of all users in the cluster subject to per-sector power constraints.

The main contributions of our scheme are listed as follows:

- Frequency reuse approach performed in the first step (inter-cluster level) can effectively reduce the inter-cluster interference for CEU. Moreover, the user partition and the frequency partition are performed at the first step. In this way, only a subset of users is mapped to each subchannel, leading to a significant reduction of both the feedback requirements and the computational complexity in the second step of the proposed scheme.

- The proposed two-step resource allocation scheme can be implemented in different time scales, i.e., the inter-cluster interference mitigation would be more static than the intra-cluster scheduling and power allocation performed in the second step. Moreover, radio resource allocation is independently performed in each cluster without inter-cluster information exchange. Therefore, no inter-cluster coordination links are needed, which is an attractive property for the deployment of practical systems.

The proposed resource allocation scheme is compared with the universal frequency reuse (UFR) scheme and the inter-cluster interference pre-cancellation (IPC) strategy proposed in [14]. Simulation results demonstrate that a significant improvement on both the celledge and the cell-average performance can be obtained by the proposed scheme, with a much lower computational complexity.

The rest of the article is organized as follows: Section 2 describes the system model and introduces the problem formulation for the downlink of a clustered network MIMO OFDMA system. In Section 3, two cooperative frequency reuse approaches are proposed for mitigating inter-cluster interference. Then, for the intra-cluster level, a joint scheduling and power allocation algorithm is proposed for each cluster in Section 4. Section 5 presents the system level simulation results. Conclusions and future work are drawn in Section 6.

Notations: $|\cdot|$ denotes the cardinality of a set. $\|\cdot\|$ denotes the Euclidean norm of a vector or absolute value of a scalar. $(\cdot)^{T}$ and $(\cdot)^{*}$ denote the transpose operation and conjugate transpose operation, respectively.

\section{System model and problem formulation}

Consider the downlink of a network MIMO OFDMA system with multiple 3-sector BSs. Each sector has one directional antenna, and it is associated with a directional cell area. The three antennas of each BS are located at the same site. Each user is equipped with one receive antenna and assigned to a serving sector that is selected based on long-term channel gain, i.e., pathloss and shadow fading. The whole system is statically divided into a number of disjoint clusters, where each cluster consists of three neighboring sectors belonging to different BSs. An example of a coordinated cluster is illustrated in Figure 1.

Assume that all the clusters have the same number of sectors $B$. Each sector has the same $N$ subchannels, i.e., there are $N$ subchannels available for joint transmission per cluster. Every cluster is working independently in the system. Without loss of generality, we consider that a given cluster is denoted by $c$ with $K^{c}$ users. Each of the sectors in cluster $c$ is denoted by $(c, b)$, where $b \in$ $\{1, \ldots, B\}$. Concentrating on an arbitrary time slot, the sectors within cluster $c$ provide joint transmission for the scheduled users (denoted by $S(c, n)$ ) on each subchannel $n$ based on the available CSI. The received signal of the scheduled user $k$ on subchannel $n$ in cluster $c$ is given as 


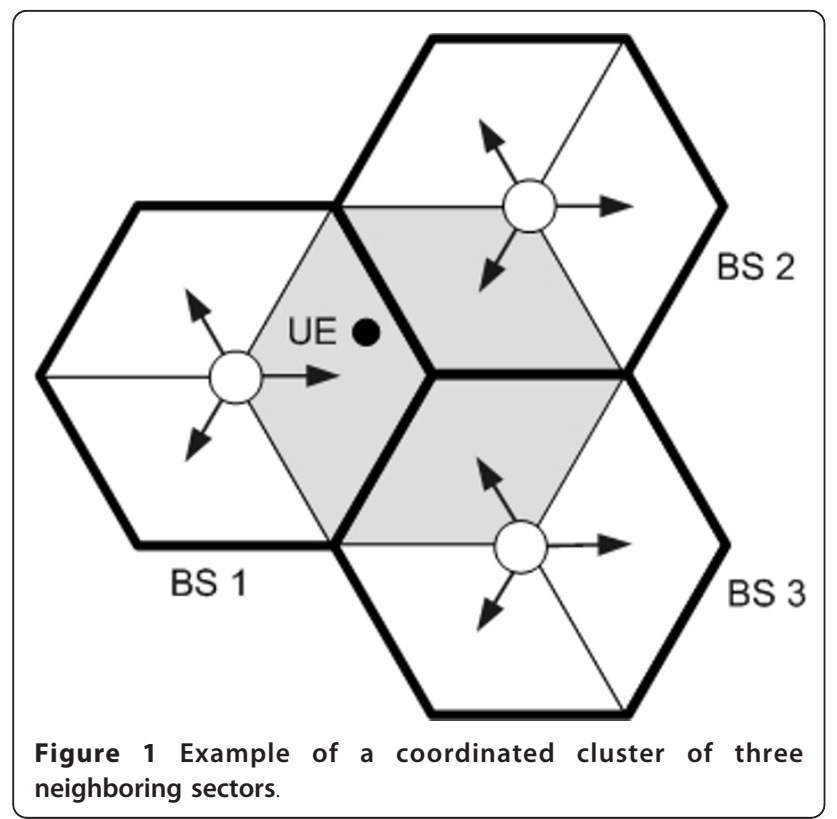

$$
\begin{aligned}
y_{k, n}^{c} & =\underbrace{\sum_{b=1}^{B} h_{k, n}^{(c, b)} w_{k, n}^{(c, b)} x_{k, n}^{c}}_{\text {desired signal }} \\
& +\underbrace{\sum_{b=1}^{B} h_{k, n}^{(c, b)} \sum_{i \in S(c, n), i \neq k} w_{i, n}^{(c, b)} x_{i, n}^{c}}_{\text {intra-cluster interference }} \\
& +\underbrace{\sum_{\hat{c} \neq c} \sum_{\hat{b}=1}^{B} h_{k, n}^{(\hat{c}, \hat{b})} \sum_{j \in S(\hat{c}, n)} w_{j, n}^{(\hat{c}, \hat{b})} x_{j, n}^{\hat{c}}}_{\text {intra-cluster interference }}+z_{k, n},
\end{aligned}
$$

where $h_{k, n}^{(c, b)}$ denotes the complex channel response between sector $(c, b)$ and user $k$ on subchannel $n$, consisting of path loss, shadow fading, and small-scale fading. $w_{k, n}^{(c, b)}$ is the beam-forming weight for user $k$ on subchannel $n$ with respect to sector $(c, b) . x_{k, n}^{c}$ denotes the data symbol for user $k$ on subchannel $n$, which is transmitted from all the sectors inside cluster c. $z_{k, n}$ is the additive white Gaussian noise at user $k$ on subchannel $n$ with zero mean and variance $\sigma^{2}$.

Let $\mathbf{h}_{k, n}^{c}=\left[h_{k, n}^{(c, 1)}, \ldots, h_{k, n}^{(c, B)}\right]$ and $\mathbf{w}_{k, n}^{c}=\left[w_{k, n}^{(c, 1)}, \ldots, w_{k, n}^{(c, B)}\right]^{T}$ denote the channel vector and the beamforming vector from all sectors in cluster $c$ to user $k$ on subchannel $n$, respectively. The maximum number of users that can be supported on subchannel $n$ in cluster $c$ is bounded by the total number of transmit antennas of cluster $c$, i.e., | $S(c, n) \mid \leq B$. In this article, zero-forcing joint transmission is used to eliminate the intra-cluster interference.
The beamforming matrix is defined as the pseudoinverse of the channel matrix. Thus, we have

$$
\mathbf{h}_{k, n}^{c} \mathbf{w}_{i, n}^{c}=\left\{\begin{array}{l}
0, i \in S(c, n), i \neq k \\
1, i \in S(c, n), i=k .
\end{array}\right.
$$

Then, the received signal $\gamma_{k, n}^{c}$ becomes

$$
y_{k, n}^{c}=\underbrace{x_{k, n}^{c}}_{\text {desired signal }}+\underbrace{\sum_{\hat{c} \neq c} \sum_{b=1}^{B} h_{k, n}^{(\hat{c}, \hat{b})} \sum_{j \in S(\hat{c}, n)} w_{j, n}^{(\hat{c}, \hat{b})} x_{j, n}^{\hat{c}}}_{\text {inter - cluster interference }}+z_{k, n} .
$$

Denote $p_{k, n}^{c}=x_{k, n}^{c} x_{k, n}^{c *}$ as the symbol power allocated to user $k$ on subchannel $n$ across the $B$ sectors in cluster $c$. Then, the signal to interference plus noise ratio (SINR) of user $k$ on subchannel $n$ is derived as

$$
\gamma_{k, n}=\frac{p_{k, n}^{c}}{\sum_{\hat{c} \neq c} \sum_{j \in S(\hat{c}, n)}\left\|\mathbf{h}_{k, n}^{\hat{c}} \mathbf{w}_{j, n}^{\hat{c}}\right\|^{2} p_{j, n}^{\hat{c}}+\sigma^{2}} .
$$

Finally, based on the Shannon theorem, the achievable transmission rate of user $k$ on subchannel $n$ can be expressed as

$$
R_{k, n}=W \log _{2}\left(1+\beta \gamma_{k, n}\right),
$$

where $W$ is the bandwidth of each subchannel, and $\beta$ is the SINR gap, which is a constant related to the target bit error rate (BER) given as $\beta=-1.5 / \ln (B E R)$ using $M-$ QAM modulation [30]. Then, the instantaneous data rate for user $k$ at a given time slot becomes

$$
R_{k}=\sum_{n=1}^{N} R_{k, n}=W \sum_{n=1}^{N} \log _{2}\left(1+\beta \gamma_{k, n}\right) .
$$

The transmit power of sector $(c, b)$ on subchannel $n$ is given by

$$
P_{n}^{(c, b)}=\sum_{k \in S(c, n)}\left\|w_{k, n}^{(c, b)}\right\|^{2} p_{k, n^{\prime}}^{c}
$$

and the total transmit power of sector $(c, b)$ is

$$
P^{(c, b)}=\sum_{n=1}^{N} P_{n}^{(c, b)}=\sum_{n=1}^{N} \sum_{k \in S(c, n)}\left\|w_{k, n}^{(c, b)}\right\|^{2} p_{k, n}^{c} .
$$

In this article, the maximum available transmit power at each sector is restricted to a $P_{\max }^{(c, b)}$ value, that is, $P^{(c, b)} \leq P_{\max }^{(c, b)}$ for sector $(c, b)$.

Targeting practical scenarios, radio resource allocation is independently performed in each cluster. The objective is to maximize the sum utility of all users in the cluster under per-sector power constraints. For any given time slot, the coordinated sectors within each 
cluster can jointly determine (1) the set of users scheduled on each subchannel, and (2) the symbol power allocated to each scheduled user.

Concentrate on one arbitrary cluster $c$ with $K^{c}$ users. Let $U_{k}(\cdot)$ denote the utility function of user $k$, which is assumed to be continuously differentiable, non-decreasing and concave to balance the efficiency and fairness of the system performance. Let $\mathbf{P}^{c}=\left[p_{k, n}^{c}\right]$ denote the $K^{c} \times$ $N$ sized symbol power allocation matrix in a scheduling interval, and $\mathbf{S}^{c}=[S(c, n)]$ denote the selected user sets on each subchannel. Then, the objective function of maximizing the sum utility of all users under per-sector power constraints can be formulated as

$$
\max U\left(\mathbf{P}^{c}, \mathbf{S}^{c}\right)=\sum_{k=1}^{K^{c}} U_{k}\left(\bar{R}_{k}(t)\right) .
$$

The average data rate of user $k$ at time slot $t$ is updated using an exponentially low-pass time window as [31]

$$
\bar{R}_{k}(t)=(1-\rho) \bar{R}_{k}(t-1)+\rho R_{k}(t),
$$

where $\rho=\left(T_{s} / T_{w}\right), T_{s}$ is the slot length, and $T_{w}$ is the length of the window. $R_{k}(t)$ is the instantaneous data rate for user $k$ at a time slot $t$ and can be derived by (6). Then, based on the first-order Taylor expansion, the objective function in (9) can be rewritten as

$$
\max U\left(\mathbf{P}^{c}, \mathbf{S}^{c}\right)=\sum_{k=1}^{K^{c}} U_{k}^{\prime}\left(\bar{R}_{k}(t-1)\right) R_{k}(t),
$$

which can be interpreted as maximizing the weighted sum rate, as $U_{k}^{\prime}\left(\bar{R}_{k}(t-1)\right)$ is fixed at time slot $t$. From now on, $\mu_{k}$ is used to represent $U_{k}^{\prime}\left(\bar{R}_{k}(t-1)\right)$. It should be pointed out that the first order Taylor expansion approximation is sub-optimal. However, this approximation relaxes the original complex optimization problem to a weighted sum rate maximization problem, which greatly simplifies the algorithm design. The weights are adaptively controlled by the marginal utility with respect to the current average rates. Specifically, as has been analyzed in [31], if the utility function is defined as a natural logarithm of the user's average data rate at the current time slot, the objective becomes to maintain proportional fairness among users. Therefore, the utility-based algorithm presented by the first order Taylor expansion approximation can be treated as a general framework for allocating multi-user shared resources.

Substituting (6) and (8) into (11), the optimization problem under per-sector power constraints can be expressed as

$$
\begin{aligned}
& \max U\left(\mathbf{P}^{c}, \mathbf{S}^{c}\right)=\sum_{n=1}^{N} \sum_{k \in S(n)} \mu_{k} W \log _{2}\left(1+\beta \gamma_{k, n}\right) \\
& \text { s.t. } \sum_{n=1}^{N} \sum_{k \in S(n)}\left\|w_{k, n}^{b}\right\|^{2} p_{k, n}^{c} \leq P_{\max }^{(c, b)}, \quad b \in\{1, \ldots, B\},
\end{aligned}
$$

Note that the overall resource allocation problem is a non-convex combinatorial optimization problem. In addition, to solve the overall optimization problem, a significant amount of CSI feedback and information exchange between clusters is needed. Thus, computing its optimal solution within one step would require global network coordination, which is not realistic for implementation in real systems. Therefore, we propose a two-step resource allocation scheme to get closer to a practical implementation. In the following, we focus on a system with $B=3$. The proposed scheme can be easily extended to the $B>3$ case, e.g., multiple neighboring clusters can be grouped together to form a new bigger cluster.

\section{Inter-cluster interference mitigation}

As shown in Section 2, the intra-cluster interference can be completely eliminated by joint transmission as long as $|S(c, n)| \leq B$. However, since the neighboring clusters are also using the same $N$ subchannels, a cluster of coordinated sectors still causes inter-cluster interference to the users in the neighboring clusters, especially to the users in the cluster-edge area.

In this section, based on the idea of static fractional frequency reuse, two cooperative frequency reuse approaches are proposed to mitigate the inter-cluster interference. These frequency reuse approaches will be considered as the first step or inter-cluster level of the proposed resource allocation scheme.

\subsection{Cooperative frequency reuse Scheme 1}

From a cluster-specific point of view, users in each cluster can be divided into two classes, that is, CEU and CCU. In [14], the authors propose a user partition method based on user locations, and determine an inter-cluster coordination area by a predefined coordination distance. However, this distance parameter based user partition could be a hard decision for a real implementation. In addition, the effect of shadow fading on the users is ignored. In $[28,29]$, the user partition is instead based on the long term channel gain, which is more suitable for a practical use. Since the clusters are overlapping in $[28,29]$, the partition is performed from a cell-specific point of view, where users in each cell are divided into cell-center users and cell-edge users. In this article, we propose a clusterspecific user partition approach based on the long term channel gain, which is defined as follows. 
Definition: User partition threshold, $\Delta l$, is the threshold used for classifying CEU and CCU. User $k$ in sector $(c, b)$ estimates and feeds back to its serving sector the long term channel gains from its serving sector and from four candidate neighboring sectors, that is, the two neighboring sectors within the same cluster $c$, and the other two neighboring sectors within the BS where its serving sector belongs to. In the example of Figure 1, the measurement set for the UE consists of its serving sector, the two neighboring sectors belonging to BS1 and the two neighboring sectors belonging to its coordinated cluster (the shadowed area). After obtaining these values, cluster $c$ finds out the weakest long term channel gain within the cluster (denoted by $l_{k}^{\text {in }}$ in $\mathrm{dB}$ ) and the strongest long term channel gain from the two candidate neighboring sectors outside the cluster (denoted by $l_{k}^{\text {out }}$ in $\mathrm{dB}$ ). Note that $l_{k}^{\text {in }}$ reflects the weakest link within the cluster, which is the dominant link that affects the performance gain provided by intra-cluster zero-forcing joint transmission [32]. $l_{k}^{\text {out }}$ reflects the strongest interference link outside the cluster. If $l_{k}^{\text {out }}-l_{k}^{\text {in }} \geq \Delta l$, inter-cluster interference would compromise the intracluster joint transmission gain, i.e., inter-cluster interference would be a big challenge for user $k$. Hence, user $k$ is considered as a CEU if $l_{k}^{\text {out }}-l_{k}^{\text {in }} \geq \Delta l$; otherwise, it is regarded as a CCU. The threshold value can be predefined by each cluster or by the network (as employed in the handoff algorithm for practical wireless networks), and it can be a parameter to optimize according to the network design objective. Note that the measurements required from the users are based on the long term channel gain, which can be obtained from the ones used for the handoff process [33]. Hence, there is no measurements and feedback increase from the users by using this user partition method. One approach to further reduce the feedback would be to obtain $l_{k}^{\text {in }}$ and $l_{k}^{\text {out }}$ at the user side. Hence, these values or $l_{k}^{\text {out }}-l_{k}^{\text {in }}$ could be instead fed back for user partition.

The $N$ frequency subchannels are divided into two orthogonal sets, $G$ and $F$, where $F$ is further divided into three orthogonal subsets, marked by $f_{i}$, with $i=\{1,2,3\}$. Subchannels in set $G$ are used for CCU with frequency reuse factor of one for each cluster, while subchannels in set $F$ are used for CEU with frequency reuse factor of $1 / 3$ for each cluster. This cooperative frequency reuse scheme, named as CFR-1, is shown in Figure 2. Note that neighboring clusters adopt orthogonal subchannels for the CEU. Hence, the inter-cluster interference can be significantly reduced. The frequency partition (the size of the cluster-center frequency set $G$ ) can be a parameter to optimize, and it is treated by system level simulation in Section 5.

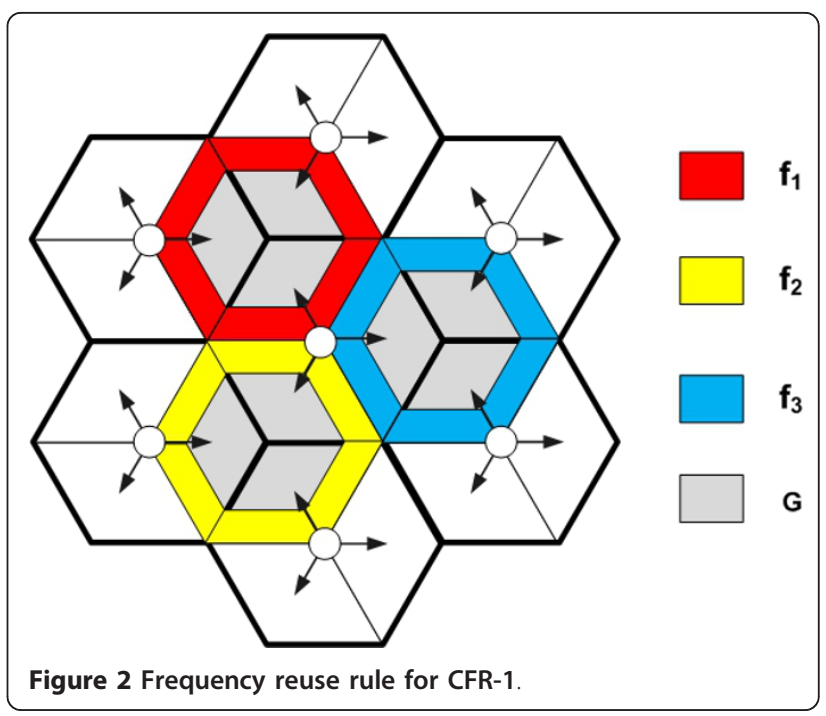

\subsection{Cooperative frequency reuse Scheme 2}

In CFR-1, the frequency reuse factor for CEU is $1 / 3$, that is, only one third of the subchannels in set $F$ is available for CEU in each cluster. In this subsection, we propose a second cooperative frequency reuse scheme, named as CFR-2, where the frequency reuse factor for CEU is $2 / 3$ in each cluster.

Assume that every three neighboring clusters are grouped together and respectively marked as Clusters 1 , 2 and 3 (see Figure 3). Given the marker of each cluster (Cluster 1, 2 or 3), CEU in each cluster are further divided into two types according to their dominant interference clusters. The dominant interference cluster of user $k$ is defined as the one that the neighboring sector with the strongest long term channel gain $\left(l_{k}^{\text {out }}\right)$ belongs to. The subchannels in set $F$ are further divided into three orthogonal subsets, marked by $f_{i}$, with $i=$ $\{1,2,3\}$. Then, frequency subset $f_{i}$ is assigned for the CEU whose dominant interference cluster is cluster $i$. Based on the above defined frequency reuse rule, two subsets of $F$ are available for CEU within each cluster, where different type of CEU use the subchannels belonging to different subsets. As illustrated in Figure 3, the subchannels used for CEU in neighboring clusters in CFR-2 are not orthogonal any more. However, for each $\mathrm{CEU}$, the inter-cluster interference coming from its dominant interference cluster can be eliminated. In the example of Figure 3, the subchannels belonging to subset $f_{2}$ can be used for CEU1, when the dominant interference cluster of CEU1 is Cluster 2. Since $f_{2}$ is not available in Cluster 2 according to the frequency reuse rule of CFR-2, there will be no inter-cluster interference introduced by Cluster 2 for CEU1. 


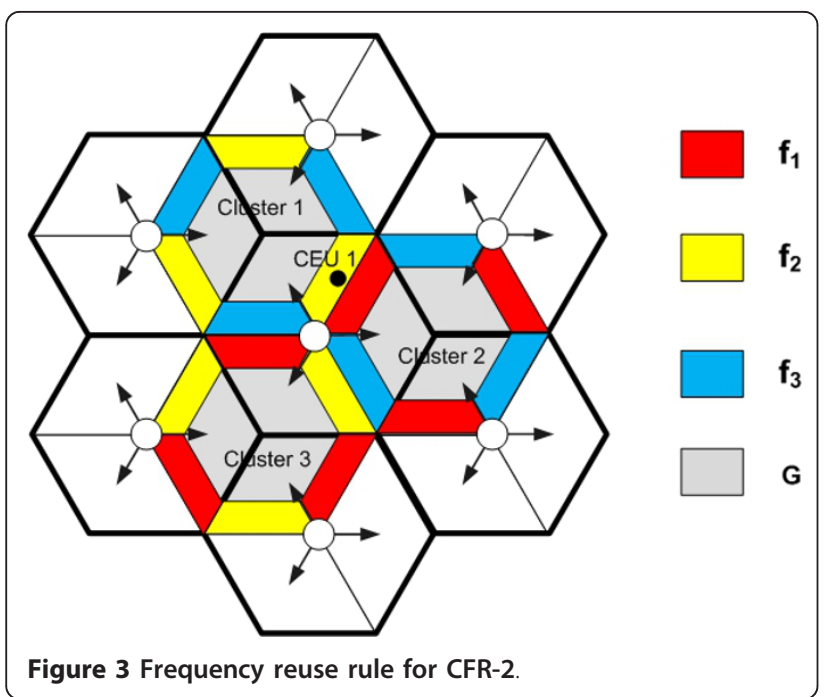

\section{Joint scheduling and power allocation}

In the inter-cluster interference mitigation or first step, users in each cluster are divided into two groups (CEU/CCU) and mapped to different frequency sets. In this section, a utility-based joint scheduling and power allocation algorithm is proposed for each cluster to solve (12), which is considered as the intra-cluster level or the second step of the proposed resource allocation scheme.

In realistic cellular systems, inter-cluster information exchange may not be feasible due to practical issues, e.g., synchronization constraints, feedback constraints, backhaul network constraints and the system complexity. Targeting practical scenarios, joint scheduling and power allocation is proposed to be independently performed in each cluster without inter-cluster communication. Assume that each cluster only has perfect CSI of the users inside this cluster, and the user data are shared by these sectors error-free and without delay. With joint transmission among the sectors within the cluster, the intra-cluster interference can be completely eliminated as long as $|S(c, n)| \leq B$. Note that the intercluster interference is reduced by frequency reuse in the first step of the resource allocation scheme. In order to avoid inter-cluster information exchange and the interdependency issues among clusters, the remaining intercluster interference is not considered in this section in the SINR expression, i.e., the signal to noise ratio (SNR) of user $k$ on subchannel $n$ in cluster $c$ based on the zero forcing joint transmission given in (4) is derived as $p_{k, n}^{c} / \sigma^{2}$. Without loss of generality, we suppress the cluster index, and concentrate on one arbitrary cluster. $b$ is used to represent $(c, b)$ to denote each of the sectors in cluster $c$. The optimization problem (12) is then given by

$$
\begin{aligned}
\max U(\mathbf{P}, \mathbf{S}) & =\sum_{n=1}^{N} \sum_{k \in S(n)} \mu_{k} W \log _{2}\left(1+\beta \gamma_{k, n}\right) \\
\text { s.t. } & \sum_{n=1}^{N} \sum_{k \in S(n)}\left\|w_{k, n}^{b}\right\|^{2} p_{k, n} \leq P_{\max , b,} \quad b \in\{1, \ldots, B\}
\end{aligned}
$$

where $\gamma_{k, n}=p_{k, n} / \sigma^{2}$, and $P_{\max , b}$ is the maximum transmit power of sector $b$. Note that the system performance could be slightly improved by redesigning a joint scheduling and power allocation algorithm where the remaining inter-cluster interference is taken into account. However, this would require an exchange of information between clusters. Moreover, the interdependency issues among clusters due to considering remaining interference would result in a system overall optimization problem, which is intractable and needs global coordination.

Equation (12) is a non-convex combinatorial optimization problem, thus, computing its globally optimal solution may not be feasible in practice. Here, we propose a suboptimal low complexity joint scheduling and power control algorithm for practical implementations. Assume $P_{\max , b}=P_{\max }$ for all sectors, and that the total transmit power $P_{\max }$ in each sector is equally pre-allocated to all the available subchannels. Then, the per-sector power constraints are reduced to per-subchannel power constraints with a constraint value $P_{\max } / N$, and the joint scheduling and power allocation problems are decoupled on each subchannel. On each subchannel $n$, equal user power allocation [3] is adopted for the users scheduled in set $S(n)$, that is, each selected user is allocated with the same symbol power given by

$$
p_{k, n}=p_{n}=\frac{P_{\max }}{\operatorname{Nmax}_{b} \sum_{k \in S(n)}\left\|w_{k, n}^{b}\right\|^{2}}, \quad k \in S(n) .
$$

It should be pointed out that equal user power allocation [3] is suboptimal, since it typically results in only one sector meeting the maximum transmitted power pre-allocated to subchannel $n$, and the remaining $B-1$ sectors transmit below the $P_{\max } / N$ value. Actually, for each subchannel $n$, the relaxed power allocation problem is a convex problem for the scheduled users in set $S(n)$. Similar to reference [19], the optimal solution can be obtained based on waterfilling distribution via standard optimization techniques. However, as mentioned in [19], the computational complexity for obtaining the optimal value is still high. For simplicity, equal user power allocation is adopted in this article.

Since we consider only SNR, i.e., $\gamma_{k, n}=p_{k, n} / \sigma^{2}$. Thus,

$$
\gamma_{k, n}=\gamma_{n}=p_{n} / \sigma^{2}, \quad k \in S(n) .
$$


Then, the sum utility of the user set $S(n)$ on subchannel $n$ can be calculated by

$$
U_{n}(S(n))=\sum_{k \in S(n)} \mu_{k} W \log _{2}\left(1+\beta \gamma_{n}\right) .
$$

Hence, under the above assumptions, the sub-optimal solution becomes an exhaustive search. For each subchannel $n$, the coordinated sectors search all possible user sets $S(n)$ in the cluster. The chosen user set $S^{*}(n)$ will be the one that achieves the highest sum utility on subchannel $n$. Since the maximum number of users that can be supported on a subchannel is bounded by the total number of transmit antennas of the cluster, i.e., $\mid S$ $(n) \mid \leq B$, the number of feasible selected user sets for each subchannel is $K^{B}$, with $K=K^{c}$ denotes the number of users in cluster $c$. Therefore, the complexity is $O(N$ $\times K^{B}$ ), which is prohibitively high. However, after the user partition and frequency partition at the first step shown in Section 3, only a subset of users is mapped to each subchannel. Therefore, the number of the feasible user sets for exhaustive search on each subchannel is reduced. The complexity is then reduced to $O\left(\sum_{i=1}^{3}\left|f_{i}\right| \times K_{i}^{B}+|G| \times K_{g}^{B}\right)$, where $K_{i}$ is the number of CEU that are mapped to the frequency subset $f_{i}$, and $K_{g}$ is the number of CCU that are mapped to the frequency subset $G$, with $\sum_{i=1}^{3}\left|f_{i}\right|+|G|=N$ and $\sum_{i=1}^{3} K_{i}+K_{g}=K$. The proposed two-step resource allocation algorithm is summarized in Algorithm 1.

\section{Simulation results}

In this section, the performance of the proposed scheme is studied by system level simulations. We consider a network MIMO OFDMA system with 19 clusters. A wrap-around technique is adopted to avoid the boundary effect, which causes the clusters in the boundary of the cellular network to receive less interference. Each cluster consists of $B=3$ neighboring sectors with one transmit antenna each. The antenna gain pattern measured in $\mathrm{dB}$ is given based on [1]

$$
A(\varphi)=-\min \left[\left(\frac{\varphi}{\varphi_{3 d B}}\right)^{2}, A_{m}\right],
$$

where $\phi$ is the angle that the user forms with the sector boresight $\phi \in\left[-180^{\circ}, 180^{\circ}\right] . \phi_{3 \mathrm{~dB}}=70^{\circ}$ is the angle associated with the half power beamwidth and $A_{m}=20 \mathrm{~dB}$ is the maximum attenuation for the sidelobe. The persector power constraint, $P_{\max }$, is $43 \mathrm{dBm}$. The cell radius is $500 \mathrm{~m}$. A typical urban multipath channel model [34] is used with path loss $L(d)=128.1+37.6 \log _{10}(d)$ in $\mathrm{dB}$, with $d$ in $\mathrm{km}$. The number of subchannels, $N$, is 50 , with each subchannel consists of 12 contiguous subcarriers. The subcarrier spacing is $15 \mathrm{kHz} . K=27$ users are uniformly dropped in each cluster with one receive antenna for each user. A full-buffer traffic model is assumed for each user. The natural logarithm function is used as the users' utility function, $U_{k}(\cdot)$. The throughput filter window length, $T_{w}$, is set to 100 time slots. The user partition threshold, $\Delta l$, is set to $-2 \mathrm{~dB}$, which will be shown as a proper choice to balance the cell-edge and cell-average data rate. This value of $\Delta l$ results in $16 \mathrm{CEU}$ and $11 \mathrm{CCU}$ in average for each cluster (over 100 different user locations). The average number of CCU and CEU per cluster $\left(K_{i}=16, K_{g}=11\right)$ will be used for calculating the complexity of the proposed Schemes 1 and 2, as shown in Table 1. Let Scheme-1 denote the proposed resource scheme adopting CFR-1 as the first step, while Scheme-2 denotes the proposed resource allocation scheme using CFR-2 as the first step. The simulation parameters are summarized in Table 2.

\subsection{Frequency partition and user partition}

In this article, we consider the following performance metrics defined in 3GPP [1]:

- Throughput cumulative distribution function (CDF) or user average data rate CDF, which is the $\mathrm{CDF}$ of the average data rate including all the users in the system.

- Cell-average user data rate, which is the $50 \%$ point of the user average data rate CDF, denoted by $R_{\text {ave }}$.

- Cell-edge user data rate, which is the $5 \%$ point of the user average data rate CDF, denoted by $R_{\text {edge. }}$

First, we consider the effect of frequency partition on the performance of Schemes 1 and 2. Assume the set of subchannels used for CEU, denoted by $F$, is equally divided into three subsets, that is $\left|f_{1}\right|=\left|f_{2}\right|=\left|f_{3}\right|=|F| / 3$, with $|F|+|G|=50$. The subchannels in set $G$ are used for CCU.

Considering different sizes of the cluster-center frequency set $G$, Figure 4 shows the CDF of the user average data rate including all CEU in the system and the CDF curves including all CCU in the system for Scheme-1. While the corresponding CDF curves for Scheme-2 are plotted in Figure 5. It can be seen that there is a tradeoff when choosing $|G|$. Actually, if $|G|$ is

Table 1 Performance comparison for different schemes

\begin{tabular}{lllll}
\hline & IPC & UFR & CFR-2 & CFR-1 \\
\hline Fairness Jug index (Mbps) & 1.60 & 2.96 & 3.32 & 6.90 \\
Complexity per cluster & 984,200 & 984,150 & 33,891 & 67,271 \\
$\begin{array}{l}\text { Inter-cluster communication } \\
\text { requirement }\end{array}$ & Yes & No & No & No \\
\hline
\end{tabular}


Table 2 Simulation parameters

\begin{tabular}{ll}
\hline Parameters & Values \\
\hline Number of clusters & 19 \\
Number of sectors per cluster, $B$ & 3 \\
Number of transmit antennas per & 1 \\
sector & \\
Maximum transmit power per sector, & $43 \mathrm{dBm}$ \\
$P_{\text {max }}$ & 27 \\
Number of users per cluster, $K^{C}$ & 1 \\
Number of receive antennas per user & 50 \\
Number of subchannels, $N$ & $180 \mathrm{kHz}$ \\
Subchannel bandwidth & $500 \mathrm{~m}$ \\
Cell radius & Typical urban [34] \\
Multipath channel model & $L(d)=128.1+37.6 \log _{10}(d), d$ in \\
Pathloss (dB) & $\mathrm{km}$ \\
Traffic model & Full buffer \\
User utility function, $U(\cdot)$ & $\ln (\cdot)$ \\
Throughput filter window length, $T_{w}$ & 100 \\
User partition threshold, $\Delta l$ & $-2 \mathrm{~dB}$ \\
\hline
\end{tabular}

large, more subchannels will be allocated to CCU, leading to CCU's performance increasing with CEU's performance decreasing for both of these two schemes. In Scheme-1, the inter-cluster interference is significantly reduced for CEU due to the use of CFR-1. The clustercenter areas are still interference limited, since the frequency reuse factor is one for the these areas. Hence, we can observe from Figure 4 that CEU achieve higher date rates compared with CCU in Scheme-1 in most cases, e.g., when $|G|<29$. In Scheme-2, the inter-cluster interference is handled by CFR-2, and CEU still suffer the interference from some neighboring clusters.
Therefore, the CEU's data rates are lower compared with that of CCU in Scheme-2, as shown in Figure 5.

In order to have a further understanding of the two proposed schemes with respect to the system performance metrics $R_{\text {edge }}$ and $R_{\text {ave }}$, the user average data rate CDF curves including all users in the system with respect to different values of $|G|$ are plotted in Figures 6 and 7 for Schemes 1 and 2, respectively. Notice that $R_{\text {edge }}$ is defined based on the user average data rate (the $5 \%$ point of the user average data rate CDF including all the users in the system) according to 3GPP [1], instead of the user category (CEU). Hence, $R_{\text {edge }}$ is not equivalent to the average date rate of CEU. For example, as shown in Figure 4, the users with lower average data rate actually come from CCU in Scheme-1. While for Scheme-2, the users with lower average data rate come from CEU as shown in Figure 5. The average date rate of $\mathrm{CCU}$ increases as the number of frequency bands allocated to the CCU $(|G|)$ increases. Hence, it is reasonable to see from Figures 6 and 7 that $R_{\text {edge }}$ increases as $|G|$ increases in Scheme- 1 , while $R_{\text {edge }}$ decreases as $\mid$ $G$ | increases in Scheme-2.

In Figure 6, there are plateau regions at $\mathrm{CDF}$ values of 0.4 and 0.93 . The plateau region at 0.4 is actually the point separating the CCU and CEU in the Scheme-1, while the plateau region at the CDF value around 0.93 comes from the CEU (see Figure 4). Based on the user partition method proposed in this article, the CEU group consists of both the users that are close to the neighboring clusters and have poor channel qualities (sector-edge users), and the users that are close to their serving sector and have good channel qualities (sectorcenter users). The CDF value around 0.93 is actually the

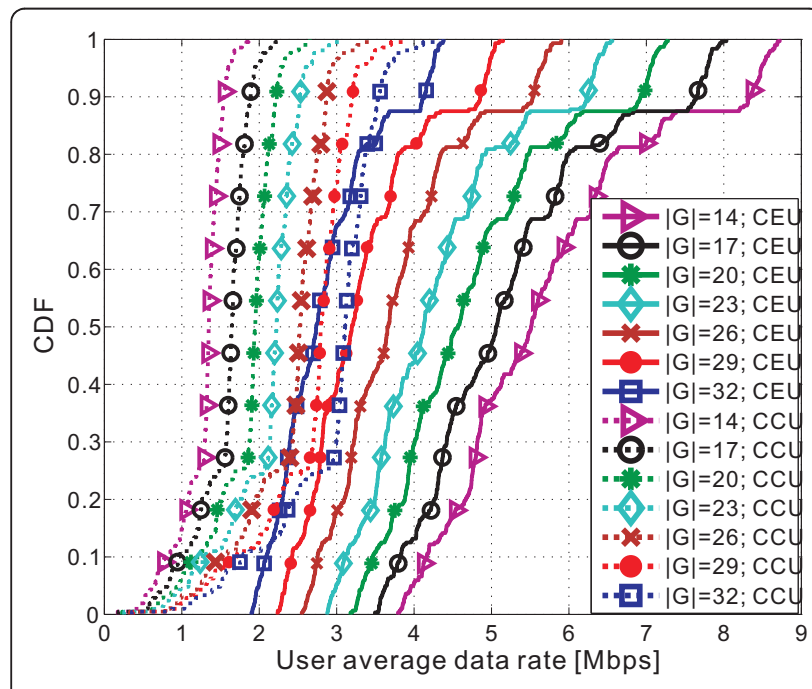

Figure $4 \mathrm{CDF}$ of user average data rate for CEU and CCU in Scheme-1.

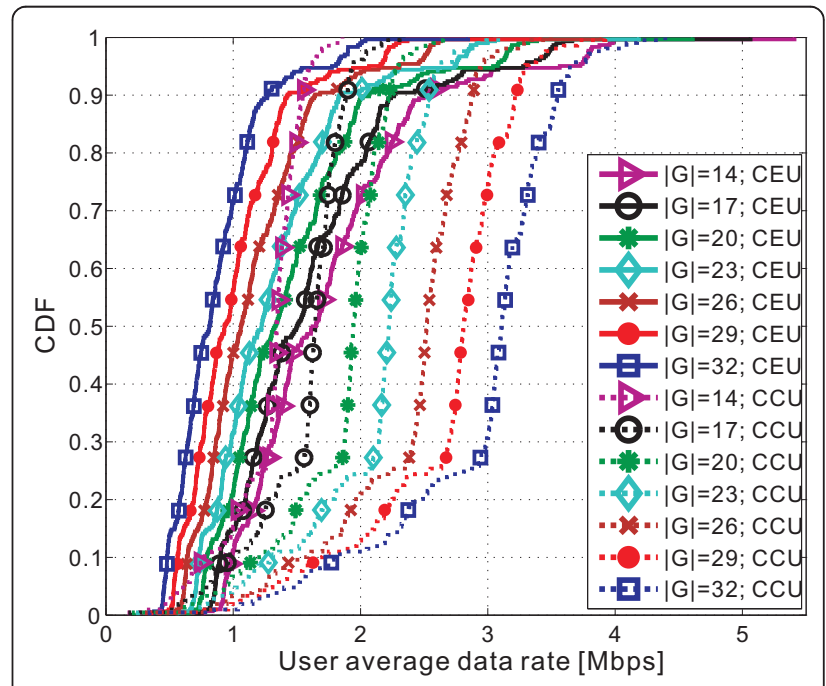

Figure 5 CDF of user average data rate for CEU and CCU in Scheme-2. 


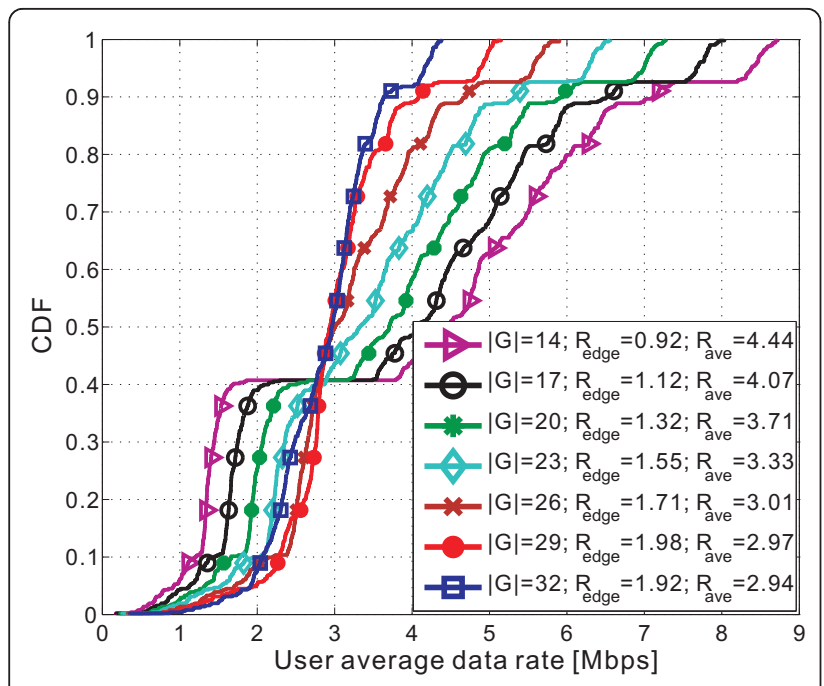

Figure 6 CDF of user average data rate in Scheme-1.

point separating the sector-edge users and the sectorcenter users within the CEU group.

The above results are obtained by setting the user partition threshold, $\Delta l$, to be $-2 \mathrm{~dB}$. Besides of frequency partition, the user partition would also affect the value of $R_{\text {edge }}$ and $R_{\text {ave. }}$. In order to balance the cell-edge and cell-average performance, a utility function is defined in [14] to evaluate the effect of coordination distance on both $R_{\text {edge }}$ and $R_{\text {ave }}$. In this article, we map the distance parameter to the size of the cluster-center frequency set $|G|$ and the user partition threshold $\Delta l$. Then, a fairness jug function with respect to the value of $|G|$ and $\Delta l$ is defined as

$$
J(|G|, \Delta l)=\alpha R_{\text {edge }}(|G|, \Delta l)+(1-\alpha) R_{\text {ave }}(|G|, \Delta l),(18
$$

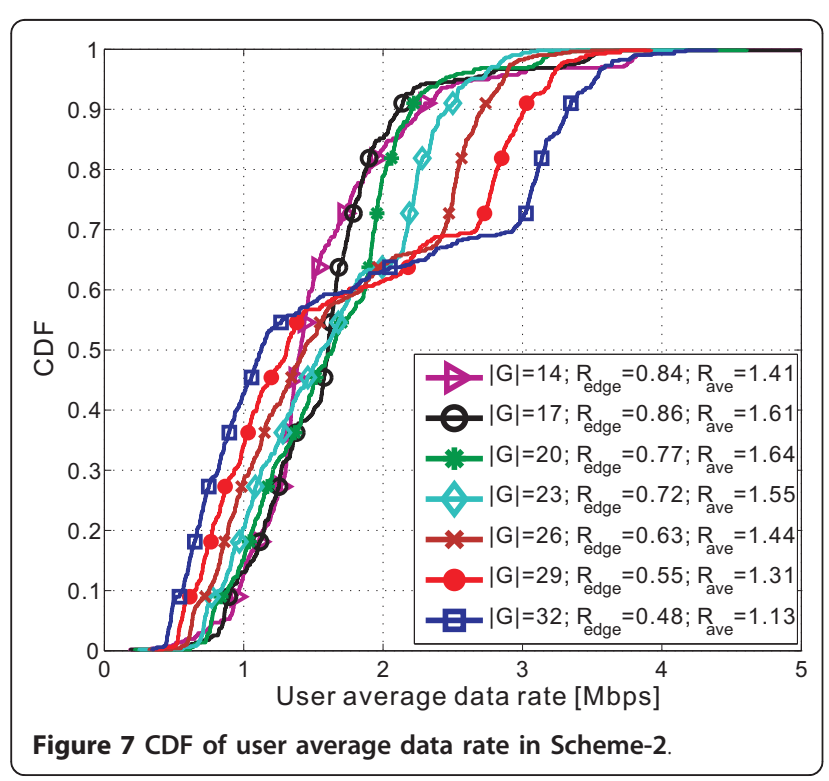

where $\alpha \in[0,1]$ is a fairness factor reflecting the design objective. When the aim is to improve the cell-edge date rate, we choose a larger value of $\alpha$. If the objective is the sum rate, a smaller value of $\alpha$ is picked. As an example, $\alpha=2 / 3$ is selected in our simulation, which means we target the cell-edge performance. Recall that a user $k$ is considered as a CEU if $l_{k}^{\text {out }}-l_{k}^{\text {in }} \geq \Delta l$; otherwise, it is a CCU. Therefore, the number of CEU decreases as the value of $\Delta l$ increases. For example, in our simulations, the percentage of CEU is around $22.2 \%$ with $\Delta l=-6 \mathrm{~dB}$, and $66.7 \%$ with $\Delta l=6 \mathrm{~dB}$.

The values of Fairness Jug Index with respect to different $|G|$ and $\Delta l$ are shown in Figures 8 and 9 for Schemes 1 and 2, respectively. For each scheme, the user average data rate CDF including all users in the system is first plotted with respect to each pair of $(|G|$, $\Delta l)$. Then, $R_{\text {edge }}(|G|, \Delta l)$ and $R_{\text {ave }}(|G|, \Delta l)$ can be obtained from the corresponding CDF curve, according to the definition in 3GPP. The Fairness Jug Index, $J(|G|$, $\Delta l$ ), is finally derived by substituting $R_{\text {edge }}(|G|, \Delta l)$ and $R_{\text {ave }}(|G|, \Delta l)$ into (18). It can be seen that the maximum values for both Schemes 1 and 2 are achieved when $\Delta l$ $=-2 \mathrm{~dB}$. While the optimal frequency partition is achieved with $|G|=29$ and $|F|=7$ for Scheme-1, $|G|=$ 17 and $|F|=11$ for Scheme-2. Compared with Scheme1 , Scheme-2 needs more subchannels for CEU. As has been mentioned above, Scheme-1 performs CFR-1 for inter-cluster interference mitigation, where neighboring clusters use orthogonal subchannels for CEU. Hence, the inter-cluster interference is significantly reduced in Scheme-1, leading to a large performance improvement for CEU (see Figure 4). In Scheme-2, CEU still suffer the interference from some neighboring clusters. Hence,

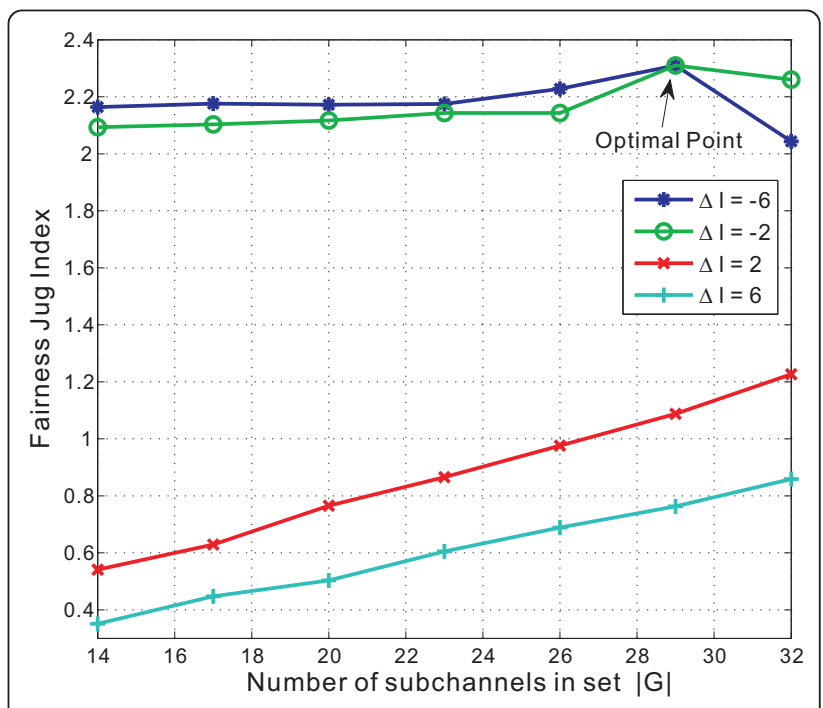

Figure 8 Fairness Jug Index in Scheme-1 with different $|G|$ and $\Delta l$. 


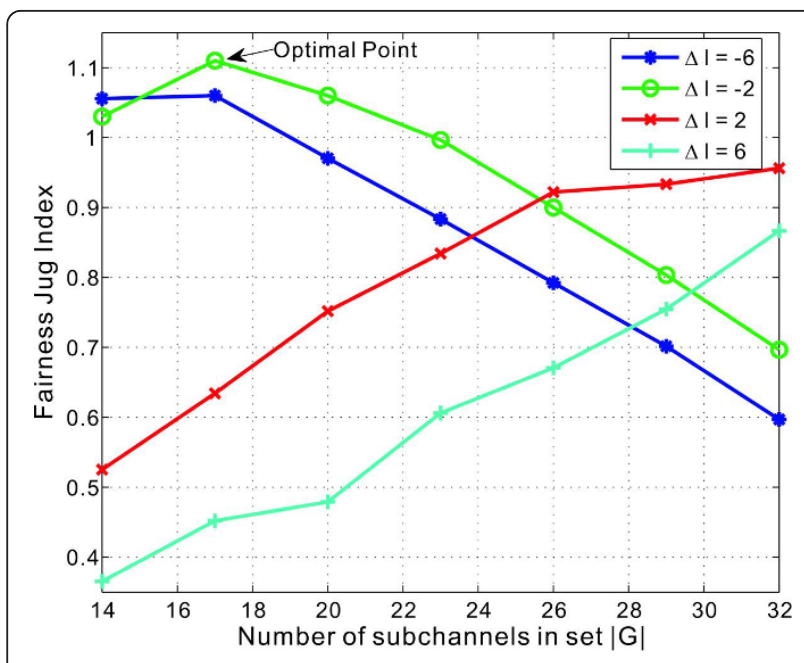

Figure 9 Fairness Jug Index in Scheme-2 with different $|G|$ and $\Delta l$.

more cluster-edge subchannels are needed in Scheme-2 to increase the user data rate of CEU (see Figure 5).

\subsection{Performance analysis}

In this simulation, based on the results from Figures 8 and 9, we choose $|G|=29$ for Scheme-1 and $|G|=17$ for Scheme-2. $\Delta l$ is set to be $-2 \mathrm{~dB}$. Besides the proposed Schemes 1 and 2, the following two schemes are considered as reference schemes for performance comparison.

- UFR, where all subchannels are available for each cluster and the proposed joint scheduling and power allocation is adopted independently within each cluster irrespective of the user category (CEU/CCU).

- IPC, where each cluster helps the neighboring CEU by taking these users into account when designing precoding matrices. In this scheme, the two-step joint scheduling approach described in [14] is adopted as follows.

- In a first step (intra-cluster level), each cluster performs joint scheduling and power allocation within its own cluster (the same approach as in the UFR scheme).

- In a second step (inter-cluster level), IPC is independently performed on each subchannel: CEU scheduled on the corresponding subchannel inform the neighboring helper clusters. Then, each cluster deals with the requests from CEU in the neighboring clusters in a sequential way, and it selects to always help those CEU by randomly dropping some of its own users scheduled on the same subchannel. After this re-scheduling process, each cluster redesigns the transmit power for the scheduled users using (14).
In Figure 10, the CDF of the average SINR of all users in the system is evaluated for the two proposed schemes. For each user, the SINR is calculated per trial, and then averaged over 100 trials. In each trial, the SINR of each user is obtained by averaging the SINR values over all subchannels that are assigned to it, considering the remaining interference power from one ring of six neighboring clusters. Note that the wrap-around technique is adopted to avoid the boundary effect for the users in the boundary clusters (the clusters in the boundary of the cellular deployment). Compared with the UFR Scheme, it is shown that the average SINR performance of the proposed schemes is significantly improved due to the inter-cluster interference reduction. Similar to the analysis for Figure 6, the plateau region at 0.4 for Scheme-1 in this figure is actually the point separating the SINR values of CCU and CEU. Due to the use of CFR-1 in Scheme-1, the inter-cluster interference reduction of CEU is quite significant, resulting in a better SINR performance for CEU compared with that of CCU in Scheme-1.

Figures 11 and 12 show the CDF of the user average data rate and the CDF of the user average utility for the considered resource allocation schemes. Table 1 gives the performance comparison of the different schemes in terms of Fairness Jug Index, computational complexity and inter-cluster communication requirements. We can see that:

- Although inter-cluster interference is treated in the IPC scheme, its performance is even worse than the UFR scheme, which does not take inter-cluster interference into account. The poor performance of the IPC scheme was caused by the following two main reasons.

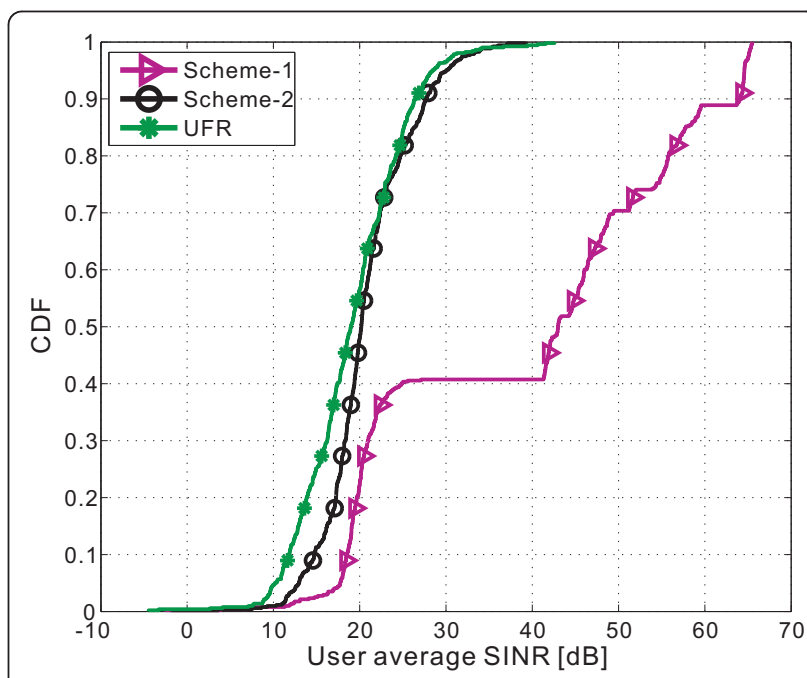

Figure $10 \mathrm{CDF}$ of user average SINR for different schemes. 


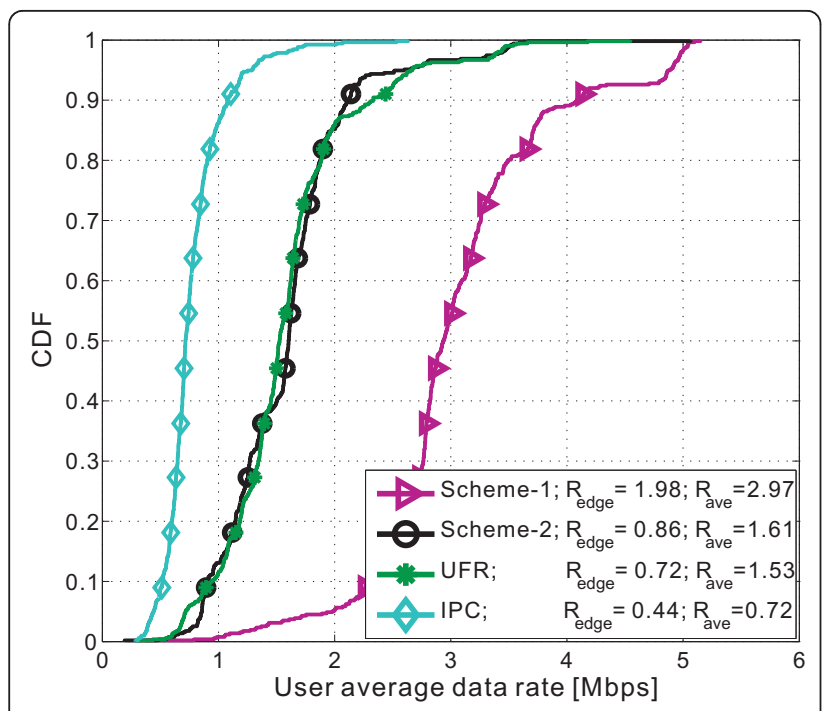

Figure $11 \mathrm{CDF}$ of user average data rate for different schemes.

- Limited degrees of freedom for each cluster to help the neighboring CEU while serving its own users. In the IPC scheme, in order to help a neighboring CEU by interference pre-cancellation, the cluster needs to provide a certain number of degrees of freedom by dropping some scheduled users of its own, which leaves fewer degrees of freedom to serve its own users. As explained in Lemma 2 of [14], for a cluster with $B$ coordinated sectors and $k_{e}$ neighboring CEU to help, the maximum number of users that can be supported simultaneously by joint transmission in this cluster is bounded by $K_{\max }^{h} \leq\left\lfloor\left(B N_{t}\right) / N_{r}\right\rfloor-k_{e}$, where $N_{t}$ denotes the number of transmit antennas per sector and $N_{r}$ denotes the number of receive

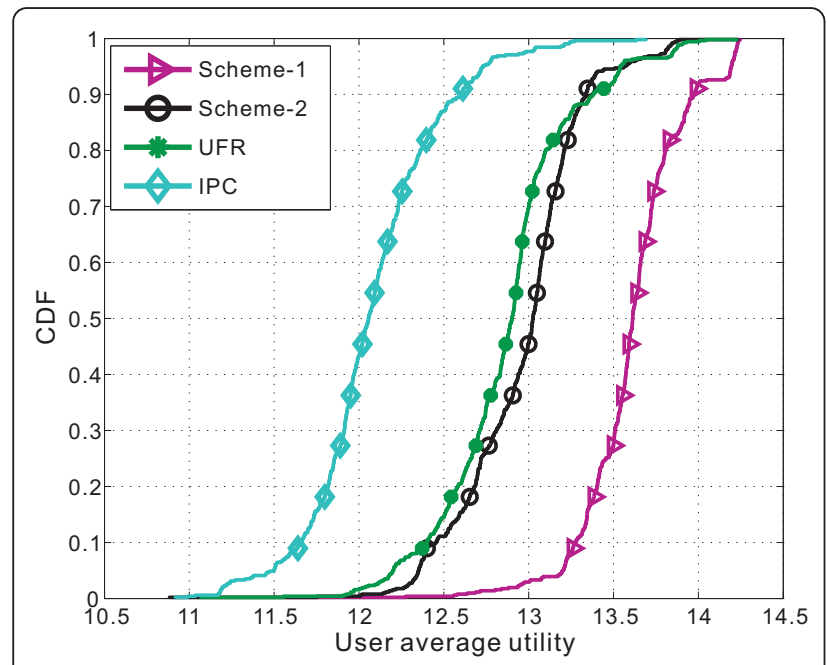

Figure $12 \mathrm{CDF}$ of user average utility for different schemes. antennas per user. Therefore, to help a neighboring CEU, the total number of users that the IPC scheme can support is reduced. In our system model, $N_{t}=N_{r}=1$. Hence, the maximum number of users that can be served within a cluster is determined by the cluster size $B$. With a larger $B$, there would be spare degrees of freedom left for each cluster to help neighboring CEU. However, note that due to path loss, the CEU do not benefit from far away sectors' transmission. As shown in [14], there is a diminishing gain with the increase of the cluster size. Therefore, the performance of Schemes 1 and 2 with respect to the IPC scheme might improve for a larger $B$.

- A smart global scheduler is required for jointly scheduling users across multiple clusters. Note that the two-step joint scheduling approach in [14] aggressively protects the neighboring CEU by randomly dropping some scheduled users of its own, which leaves fewer degrees of freedom to serve its own users. Since the dropped users in each cluster are randomly picked out, some of its own scheduled CEU might also be dropped out in Step 2, resulting in the performance degradation of CEU. In addition, for each cluster, the neighboring CEU come from six neighboring clusters. Hence, the chance that a cluster receives requests from neighboring CEU is very high. In an extreme case for a subchannel, where the number of scheduled neighboring CEU of a cluster is large (larger or equal to B), the two-step joint scheduling strategy would force the cluster to drop all its scheduled users on this subchannel to aggressively protect the neighboring CEU, leading to both CEU and CCU performance degradation. Hence, in order to improve the performance of IPC scheme, a smart global scheduler is required for joint user scheduling across multiple clusters. However, this global optimization requires intercluster communication and increases the complexity of the resource allocation design.

- In Figure 11, compared with UFR, the cell-edge user data rate of the proposed Scheme- 2 is improved by $20 \%$, while the cell-average user data rate in the Scheme- 2 is improved by $5 \%$. The proposed Scheme-1 achieves a much more significant performance improvement compared to the UFR scheme, with about $180 \%$ increase of cell-edge user data rate and $90 \%$ increase of cell-average user data rate. Note that although the average data rate and the average utility performance of Scheme- 2 is close to that of UFR scheme, the complexity of Scheme- 2 is much lower compared with UFR scheme, which can be observed in Table 1. 
- As shown in Table 1, the computational complexity of the proposed two schemes is much lower than the UFR scheme and the IPC scheme, with Scheme-2 achieving the lowest complexity. Since the inter-cluster interference mitigation approaches (CFR-1 and CFR-2) are adopted in the first step of Schemes 1 and 2 , only a subset of users is mapped to each subchannel. Hence, the number of feasible user sets for exhaustive search in the second step for Schemes 1 and 2 is significantly reduced. With $\Delta l=-2 \mathrm{~dB}$, the average numbers of CEU and CCU per cluster are $K_{i}$ $=16$ and $K_{g}=11$ respectively in our simulation. As explained in Section 4, the complexity per cluster for Schemes 1 and 2 is $O\left(\sum_{i=1}^{3}\left|f_{i}\right| \times K_{i}^{B}+|G| \times K_{g}^{B}\right)$. The number of the feasible selected user sets for each subchannel in the UFR scheme is $K^{B}$. Therefore, the complexity for the UFR scheme is $N \times K^{B}$. In the IPC scheme, each cluster performs joint scheduling and power allocation within its own cluster based on UFR in a first step, then each cluster needs to perform one more time user selection according the requests from $\mathrm{CEU}$ in the neighboring clusters. Hence, the complexity for IPC is $N \times\left(K^{B}+1\right)$. Note that the complexity of the four schemes considered in this article increases exponentially with the cluster size $B$.

\section{Conclusions}

The resource allocation problem has been considered for the downlink of a clustered network MIMO OFDMA system. A two-step resource allocation scheme with inter-cluster interference mitigation and intra-cluster joint scheduling and power allocation has been presented. In particular, the main task of managing the inter-cluster interference is accomplished by two cooperative frequency reuse approaches at the first step of the proposed resource allocation scheme. A user partition method based on the long term channel gain is introduced to divide the users of each cluster into cluster-edge and cluster-center users. Frequency subchannels in each cluster are separated into clusteredge and cluster-center frequency sets. The inter-cluster interference is reduced by mapping different groups of cluster-edge users to different subchannels of the cluster-edge frequency set in a cooperative way. We have shown that there is a tradeoff between the celledge and cell-average performance while choosing the frequency partition and the user partition, i.e., the size of the cluster-center frequency set and the user partition threshold. As the second step, a sub-optimal utility-based joint scheduling and power allocation algorithm is proposed for each cluster as the intracluster level of resource allocation. The objective is to maximize the sum utility of all users within the cluster under per-sector power constraints. Note that in realistic scenarios, the two-step approach can be implemented in different time scales, i.e., the inter-cluster interference mitigation would be more static than the intra-cluster scheduling and power allocation performed in the second step. It has been demonstrated by simulation results that our proposed resource allocation scheme can provide a considerable performance improvement in terms of the cell-edge user data rate, the cell-average user data rate and the user average utility. In addition, the proposed two-step resource allocation scheme can be implemented independently in each cluster without inter-cluster communication, which is an attractive property for practical systems, since it reduces both the network signaling overhead and the computational complexity.

In this article, we have assumed that each sector has one transmit antenna and each user has one receive antenna. Zero-forcing beamforming is used as the network MIMO joint transmission scheme. In future work, multiple antennas at both the sector side and the user side will be investigated. Frequency reuse combined with interference pre-cancellation techniques will be studied for managing the inter-cluster interference. More efficient joint scheduling and power control algorithms with advanced multi-antenna joint transmission methods will be considered. The results in this article assume that perfect CSI is available at the cluster side for the users within the cluster. Investigation of imperfect CSI is of practical importance and it is a topic of our future work.

\section{Algorithm 1. Proposed two-step resource allocation scheme \\ Step 1 Inter-cluster interference mitigation}

1: In each cluster, divide the users into $\mathrm{CCU}$ and CEU.

2: Map CCU to the subchannels in frequency set $G$.

3: Map CEU to the subchannels in frequency subset $f_{i}$ according to the predefined cooperative frequency reuse scheme (CFR-1 or CFR-2).

\section{Step 2 Joint scheduling and power allocation}

1: For each subchannel $n$, find all the users mapped to it.

2: For each feasible user set $S(n)$ on subchannel $n$ (|S $(n) \mid \leq B)$, derive the beamforming matrix by zeroforcing joint transmission.

3: Calculate the sum utility $U_{n}(S(n))$ of user set $S(n)$, based on (14), (15) and (16). 4: Find the optimal user set $S^{*}(n)$ with the maximum sum utility $U_{n}\left(S^{*}(n)\right)$, 


\section{and derive the corresponding transmit power $p_{n}^{*}$ for each user in $S^{*}(n)$ by (14).}

\begin{abstract}
Abbreviations
3GPP: 3rd generation partnership project; BER: bit error rate; BSs: base stations; CCU: cluster-center users; CDF: cumulative distribution function; CEU: cluster-edge users; CFR-1: cooperative frequency reuse Scheme 1; CFR2: cooperative frequency reuse Scheme 2; CoMP: coordinated multi-point transmission/reception; CSI: channel state information; CU: central unit; IPC: inter-cluster interference pre-cancellation; LTE: long term evolution; MIMO: multiple input multiple output; OFDM: orthogonal frequency division multiplexing; OFDMA: orthogonal frequency division multiple access; Scheme-1: resource allocation Scheme 1; Scheme-2: resource allocation Scheme 2; SINR: signal to interference plus noise ratio; SNR: signal to noise ratio; UFR: universal frequency reuse.
\end{abstract}

\section{Acknowledgements}

This work has been supported by the EU FP7 project INFSO-ICT-247223 ARTIST4G and the Swedish Agency for Innovation Systems (VINNOVA). C. Botella's work is supported by the Spanish MEC Grants CONSOLIDERINGENIO 2010 CSD2008-00010 "COMONSENS" and COSIMA TEC2010-19545C04-01. We would like to acknowledge the contributions of our colleagues. We also thank the anonymous reviewers for their useful comments.

\section{Author details}

'Department of Signals and Systems, Chalmers University of Technology, Gothenburg, Sweden ${ }^{2}$ Institute of Robotics and Information \&

Communication Technologies (IRTIC), Universitat de València, València, Spain

\section{Competing interests}

The authors declare that they have no competing interests.

Received: 20 July 2011 Accepted: 18 May 2012 Published: 18 May 2012

\section{References}

1. 3GPP TR 36.814 v9.0.0, Further Advancements for E-UTRA Physical Layer Aspects (2010)

2. G Boudreau, J Panicker, N Guo, R Chang, N Wang, S Vrzic, Interference coordination and cancellation for $4 \mathrm{G}$ networks. IEEE Commun Mag. 47(4), 74-81 (2009)

3. M Karakayali, G Foschini, R Valenzuela, Network coordination for spectrally efficient communications in cellular systems. IEEE Trans Wirel Commun. 13(4), 56-61 (2006). doi:10.1109/MWC.2006.1678166

4. H Skjevling, D Gesbert, A Hjørungnes, Low-complexity distributed multibase transmission and scheduling. EURASIP J Adv Signal Process (2008)

5. A Tölli, M Codreanu, M Juntti, Cooperative MIMO-OFDM cellular system with soft handover between distributed base station antennas. IEEE Trans Wirel Commun. 7(4), 1428-1440 (2008)

6. S Khattak, W Rave, G Fettweis, Distributed iterative multiuser detection through base station cooperation. EURASIP J Wirel Commun Netw. 2008, $1-15$ (2008)

7. C Botella, G Pinero, A Gonzalez, M De Diego, Coordination in a multi-cell multi-antenna multi-user W-CDMA system: beamforming approach. IEEE Trans Wirel Commun. 7(11), 4479-4485 (2008)

8. S Jing, DNC Tse, JB Soriaga, J Hou, JE Smee, R Padovani, Multicell downlink capacity with coordinated processing. EURASIP J Wirel Commun Netw. 2008, 1-19 (2008)

9. H Huang, M Trivellato, A Hottinen, M Shafi, PJ Smith, R Valenzuela, Increasing downlink cellular throughput with limited network MIMO coordination. IEEE Trans Wirel Commun. 8(6), 2983-2989 (2009)

10. F Boccardi, H Huang, Limited downlink network coordination in cellular networks, in Proceedings of the 18th IEEE International Symposium on Personal, Indoor and Mobile Radio Communications, Athens, Greece, pp. 1-5 (2007)

11. P Marsch, G Fettweis, A framework for optimizing the downlink performance of distributed antenna systems under a constrained backhaul, in Proceedings of the 13th European Wireless Conference, Paris, France, pp. $1-5(2007)$
12. S Venkatesan, Coordinating base stations for greater uplink spectral efficiency in a cellular network, in Proceedings of the 18th IEEE International Symposium on Personal, Indoor and Mobile Radio Communications, Athens, Greece, pp. 1-5 (2007)

13. P Marsch, G Fettweis, A decentralized optimization approach to backhaulconstrained distributed antenna systems, in Proceedings of the 16th IST Mobile and Wireless Communications Summit, Budapest, Hungary, pp. 1-5 (2007)

14. J Zhang, R Chen, JG Andrews, A Ghosh, RW Heath, Networked MIMO with clustered linear precoding. IEEE Trans Wirel Commun. 8(4), 1910-1921 (2009)

15. A Papadogiannis, D Gesbert, E Hardouin, A dynamic clustering approach in wireless networks with multi-cell cooperative processing, in Proceedings of the IEEE International Conference on Communications, Beijing, China, pp. 4033-4037 (2008)

16. F Boccardi, H Huang, A Alexiou, Network MIMO with reduced backhaul requirements by MAC coordination, in Proceedings of Asilomar Conference on Signals, Systems and Computers, Pacific Grove, California, pp. 1125-1129 (2008)

17. S Zhou, J Gong, Z Niu, Y Jia, P Yang, A decentralized framework for dynamic downlink base station cooperation, in Proceedings of the IEEE Global Telecommunications Conference, Hawaii, USA, pp. 1-6 (2009)

18. J Li, XXu, X Chen, $X$ Tao, T Svensson, C Botella, Downlink radio resource allocation for coordinated cellular OFDMA networks. IEICE Trans Commun. E93.B(4), 3480-3488 (2010)

19. A Garcia-Armada, M Sanchez-Fernandez, R Corvaja, Waterfilling schemes for zero-forcing coordinated base station transmission, in Proceedings of the IEEE Global Telecommunications Conference, Hawaii, USA, pp. 1-5 (2009)

20. B Luo, Q Cui, H Wang, X Tao, Optimal joint water-filling for coordinated transmission over frequency-selective fading channels. IEEE Commun Lett. 15(2), 190-192 (2011)

21. N Reider, A Racz, G Fodor, On scheduling and power control in multi-cell coordinated clusters, in Proceedings of the IEEE Global Telecommunications Conference, Hawaii, USA, pp. 1-7 (2009)

22. J Li, T Svensson, C Botella, T Eriksson, $X X \mathrm{Xu}, \mathrm{X}$ Chen, Joint scheduling and power control in coordinated multi-point clusters, in Proceedings of the 74th IEEE Vehicular Technology Conference, San Francisco, USA, pp. 1-5 (2011)

23. J Li, X Chen, C Botella, T Svensson, T Eriksson, Resource allocation for OFDMA systems with multi-cell joint transmission, in Proceedings of the IEEE international workshop on Signal Processing Advances in Wireless Commun, Çeşme, Turkey, pp. 1-5 (2012)

24. K Maruta, T Maruyama, A Ohta, M Nakatsugawa, Inter-cluster interference canceller for multiuser MIMO distributed antenna systems, in Proceedings of the 20th IEEE Personal, Indoor and Mobile Radio Communications, Tokyo, Japan, pp. 3079-3083 (2009)

25. S Kaviani, W Krzymien, Multicell scheduling in network MIMO, in Proceedings of the IEEE Global Telecommunications Conference, Miami, Florida, USA, pp. 1-5 (2010)

26. E Katranaras, M Imran, R Hoshyar, Sum-rate of linear cellular systems with clustered joint processing, in Proceedings of the 69th IEEE Vehicular Technology Conference, Barcelona, Spain, pp. 1-5 (2009)

27. G Caire, S Ramprashad, H Papadopoulos, C Pepin, C-EW Sundberg, Multiuser MIMO downlink with limited inter-cell cooperation: approximate interference alignmnent in time, frequency and space, in Proc 46th Annual Allerton Conference on Communication, Control, and Computing, Allerton, Chicago, pp. 730-737 (2008)

28. J Li, H Zhang, X Xu, X Tao, T Svensson, C Botella, B Liu, A novel frequency reuse scheme for coordinated multi-point transmission, in Proceedings of the 71th IEEE Vehicular Technology Conference, Taipei, Taiwan, pp. 1-5 (2010)

29. J Li, X Xu, H Zhang, X Tao, T Svensson, C Botella, X Chen, Multi-beam cooperative frequency reuse for coordinated multi-point transmission. J China Univ Posts Telecommun. 13(6), 11-18 (2010)

30. A Goldsmith, SG Chua, Variable-rate variable-power MQAM for fading channels. IEEE Trans Commun. 45(10), 1218-1230 (1997). doi:10.1109/ 26.634685

31. G Song, Y Li, Cross-layer optimization for OFDM wireless networks-part II: algorithm development. IEEE Trans Wirel Commun. 4(2), 625-634 (2005)

32. R Abildgaard Olesen, M Sternad, D Aronsson, Measurement-based evaluation of robust linear precoding for downlink CoMP, in Proc of the IEEE International Conference on Communications, Ottawa, Canada, pp. 1-6 (2012) 
33. K Dimou, M Wang, Y Yang, M Kazmi, A Larmo, J Pettersson, W Muller, $Y$ Timner, Handover within 3GPP LTE: design principles and performance, in Proceedings of the 70th IEEE Vehicular Technology Conference, Anchorage, Alaska, USA, pp. 1-5 (2009)

34. 3GPP TR 36.942 V9.1.0, Evolved Universal Terrestrial Radio Access (E-UTRA) Radio Frequency (RF) system scenarios, (2010)

doi:10.1186/1687-1499-2012-175

Cite this article as: $L i$ et al.: Resource allocation for clustered network

MIMO OFDMA systems. EURASIP Journal on Wireless Communications and Networking 2012 2012:175.

\section{Submit your manuscript to a SpringerOpen ${ }^{\circ}$ journal and benefit from:}

- Convenient online submission

- Rigorous peer review

- Immediate publication on acceptance

- Open access: articles freely available online

- High visibility within the field

- Retaining the copyright to your article

Submit your next manuscript at $\gg$ springeropen.com 